\title{
Is phosphatidylinositol 3-kinase/AKT/mammalian target of rapamycin pathway therapeutic target for esophageal adenocarcinoma
}

\author{
Kazuto Harada ${ }^{1,2}$, Shumei Song ${ }^{1}$, Hideo Baba ${ }^{2}$, Jaffer A. Ajani ${ }^{1}$ \\ ${ }^{1}$ Department of Gastrointestinal Medical Oncology, University of Texas M. D. Anderson Cancer Center, Houston, TX, USA; ${ }^{2}$ Department of \\ Gastroenterological Surgery, Graduate School of Medical Science, Kumamoto University, Kumamoto, Japan \\ Correspondence to: Jaffer A. Ajani, MD. Department of Gastrointestinal Medical Oncology, University of Texas M. D. Anderson Cancer Center, 1515 \\ Holcombe Blvd, FC10.3022, Houston, TX 77030, USA. Email: jajani@mdanderson.org. \\ Provenance: This is an invited Editorial commissioned by Editor-in-Chief Changqing Pan (Shanghai Chest Hospital Affiliated to Shanghai Jiao Tong \\ University, Shanghai, China). \\ Comment on: Zaidi AH, Kosovec JE, Matsui D, et al. PI3K/mTOR Dual Inhibitor, LY3023414, Demonstrates Potent Antitumor Efficacy Against \\ Esophageal Adenocarcinoma in a Rat Model. Ann Surg 2017;266:91-8.
}

Received: 10 October 2017; Accepted: 17 October 2017; Published: 08 November 2017.

doi: $10.21037 /$ shc.2017.10.07

View this article at: http://dx.doi.org/10.21037/shc.2017.10.07

\section{Introduction}

Esophageal cancer (EC) is the eleventh most common cause of cancer worldwide $(459,299$ cases) and the sixth most common cause of cancer mortality (439,000 deaths) (1). Esophageal adenocarcinoma (EAC) is one of the major histological types of $\mathrm{EC}$ with the rapidly rising incidence in the West. Molecularly targeted drugs that have produced modest advantage in EAC patients include trastuzumab (for patients with HER2 positive EAC) but VEGFR2 inhibitor, ramucirumab in combination with paclitaxel in the secondline setting $(2,3)$. Other molecular biomarkers to select therapy are PD-L-1 and MSI status. However, data are preliminary and need to be pursued.

Zaidi and colleagues recently published a report in which they assessed the efficacy of a phosphatidylinositol 3 -kinase $(\mathrm{PI} 3 \mathrm{~K}) / \mathrm{mammalian}$ target of rapamycin (mTOR) dual inhibitor, LY3023414, on established EAC in vivo model (4). They used the Levrat's surgical model of esophagojejunostomy in rats, which leads to gastroduodenal esophageal reflux and subsequent development of reproducible EAC in $70 \%$ of rats at 28 weeks. Thirty two weeks after Levrat's surgery, rats were randomized to one of the two groups; LY3023414 treatment cohort and control cohort. Tumors volume evaluation by magnetic resonance imaging (MRI) 8 weeks after randomization showed that tumor in control group increased $109.2 \%$, and tumor in LY3023414 group decrease $56.8 \%$. Moreover, 5 of the 13 treatment cohort achieved complete tumor response by MRI. Other 8 tumors had histological evaluation, which showed that 3 achieved complete histological response and 3 had more than $50 \%$ histological response. In addition, PI3K/v-AKT murine thymoma viral oncogene homolog (AKT)/mTOR pathway and Ki67 (proliferation maker) was significantly decreased in the treated tumors, while cleaved caspase-3 (apoptosis maker) was significantly increased in the treated tumors compared to control tumors. These preclinical model results suggest that inhibition of $\mathrm{PI} 3 \mathrm{~K} / \mathrm{AKT} / \mathrm{mTOR}$ pathway is possibly effective for EAC by impairing proliferation and facilitating apoptosis.

\section{PI3K/AKT/mTOR pathway alternation and clinical outcome}

TCGA recently reported an integrated genomic landscape in EAC and show that EAC were similar with chromosomal instability (CIN) subtype for gastric cancer characterized by TP53 mutation, ERBB2 amplification, and VEGFA amplification (5). This result is consistent with clinical benefit of HER2 inhibitor and VEGFR2 inhibitor for EAC. 
TCGA research also showed that $13 \%$ EAC have PI3K/ AKT/MTOR pathway alteration with PIK3CA activation (3\%), PIK3R1 inactivation (3\%), and phosphatase and tensin homolog (PTEN) inactivation (7\%) (5). Another study of whole-exome sequencing with 149 EACs also showed that the PI3K pathway was altered in $13 \%$ by PIK3CA (6\%), PIK3R1 (4\%) and PTEN (3\%) mutation (6). Moreover, Prins et al. performed Immunohistochemistry assay and showed that p-mTOR overexpression was detected in $19.7 \%$ and correlated with poor survival (7). These results suggest that approximately $20 \%$ EAC might be driven primarily by the PI3K/AKT/MTOR pathway. However, the relationship between PIK3CA mutation and survival remains unclear for EAC. Shigaki et al. showed that PIK3CA mutation was associated with better survival in esophageal squamous cell carcinoma (8), while Harada et al. showed that PIK3CA mutation was not associated with survival in gastric cancer (9). In addition, the PI3K/AKT/ mTOR pathway activation confers treatment resistance. Hildebrandt et al. demonstrated that genetic variations in genes which were related to PI3K/AKT/MTOR pathway correlated with increased recurrence risk in EAC patient who underwent therapy (10). Saeed et al. demonstrated that AKT expression level involved in pathologic response to preoperative chemoradiation for EAC; non-responders had higher expressions of AKT compared to complete responders (11). Therefore, the $\mathrm{PI} 3 \mathrm{~K} / \mathrm{AKT} / \mathrm{mTOR}$ pathway inhibition might be effective for EAC activating $\mathrm{PI} 3 \mathrm{~K} / \mathrm{AKT} / \mathrm{mTOR}$ pathway.

\section{mTOR inhibitor for EAC}

An mTOR inhibitor, everolimus, was evaluated in the GRANITE-1 study, which compared everolimus vs. placebo in advanced gastric cancer, and did not benefit overall survival (12). There is another study that compared paclitaxel plus everolimus/placebo but that trial also did not provide advantage (NCT01248403) (13). However, several shortcomings should be considered. Firstly, eligible patients had no evaluation of PI3K/AKT/ mTOR pathway activation before treatment. Therefore, efficacy of mTOR inhibitor for GAC with activated $\mathrm{PI} 3 \mathrm{~K} / \mathrm{AKT} / \mathrm{mTOR}$ pathway remains unclear. Secondly, mTOR inhibition upregulates phosphorylation of AKT, resulting in resistance for mTOR inhibitor (14). Finally, as everolimus target only complex mTORC1, mTORC2 continue to activate downstream. Thus, dual targeted inhibitor, such as LY3023414, potentially overcomes this resistant mechanism.

\section{Combination with ERK-MAPK pathway inhibition}

The PI3K/AKT/mTOR pathway inhibitor in combination ERK-MAPK pathway inhibitor are found to be more effective rather than single pathway inhibition (15). The two pathways have a complex cross-talk, leading to positive or negative feedback in various contexts (16). MEK inhibition upregulates PI3K pathway signaling, causing resistance to MEK inhibition (17). Tumors harboring RAS or RAF mutation, for example colon cancer, is resistant for single use of growth factor receptor inhibitor (18). The PI3K/AKT pathway was found out to be activated by BRAF inhibitors in BRAF mutated colon cancer, and thus the combination therapy with BRAF inhibitors and PI3K inhibitor has been evaluated in ongoing trial (19). However, EAC rarely have RAS or RAF mutation $(5,6)$.

\section{Potential for trastuzumab resistance}

Resistant EAC for current standard treatment is the big issue to be overcome. Deguchi et al. showed that PTEN loss were more frequently detected in gastroesophageal adenocarcinoma with HER2-overexpression than that without HER2-overexpression, and PTEN loss caused a poor response to trastuzumab based therapy (20). Given that PTEN loss activates PI3K/AKT/mTOR pathway, this result suggests that HER2 inhibitor in combination with $\mathrm{PI} 3 \mathrm{~K} / \mathrm{AKT} / \mathrm{mTOR}$ pathway inhibitor might be effective for tumor with HER2 inhibitor resistance.

\section{Limitations of the mice studies}

We congratulate the authors for an excellent study. They have a successful animal model and completed pharmacodynamic studies in addition to efficacy studies. However, all preclinical studies have limitations. The preclinical models have consistently have come up short in predicting benefits in the clinic. The Zaid et al.'s paper helps us to move forward. We need better models where humanderived cancer tissue can be evaluated.

\section{Conclusions}

In summary, the PI3K/AKT/mTOR pathway involved in promoting cancer cell proliferation for EAC, thus its inhibitor might be effective for selected EAC patients. 
$\mathrm{PI} 3 \mathrm{~K} / \mathrm{AKT} / \mathrm{mTOR}$ pathway inhibition with multi target or ERK-MAPK pathway inhibition might have a potential. Further study is needed for clinical use.

\section{Acknowledgements}

Funding: This research was supported by generous grants from the Caporella, Dallas, Sultan, Park, Smith, Frazier, Oaks, Vanstekelenberg, Planjery, and Cantu families, as well as from the Schecter Private Foundation, Rivercreek Foundation, Kevin Fund, Myer Fund, Dio Fund, Milrod Fund, and The University of Texas MD Anderson Cancer Center (Houston, Texas, USA) multidisciplinary grant program. This research was also supported in part by the National Cancer Institute and Department of Defense awards CA138671, CA172741, CA129926, CA150334 (JA Ajani), and by a grant from the Japan Society for the Promotion of Science Overseas Research Fellowships (K Harada).

\section{Footnote}

Conflicts of Interest: The authors have no conflicts of interest to declare.

\section{References}

1. Global Burden of Disease Cancer C, Fitzmaurice C, Allen C, et al. Global, Regional, and National Cancer Incidence, Mortality, Years of Life Lost, Years Lived With Disability, and Disability-Adjusted Life-years for 32 Cancer Groups, 1990 to 2015: A Systematic Analysis for the Global Burden of Disease Study. JAMA Oncol 2017;3:524-48.

2. Bang YJ, Van Cutsem E, Feyereislova A, et al. Trastuzumab in combination with chemotherapy versus chemotherapy alone for treatment of HER2-positive advanced gastric or gastro-oesophageal junction cancer (ToGA): a phase 3, open-label, randomised controlled trial. Lancet 2010;376:687-97.

3. Wilke H, Muro K, Van Cutsem E, et al. Ramucirumab plus paclitaxel versus placebo plus paclitaxel in patients with previously treated advanced gastric or gastro-oesophageal junction adenocarcinoma (RAINBOW): a double-blind, randomised phase 3 trial. Lancet Oncol 2014;15:1224-35.

4. Zaidi AH, Kosovec JE, Matsui D, et al. PI3K/mTOR Dual Inhibitor, LY3023414, Demonstrates Potent Antitumor Efficacy Against Esophageal Adenocarcinoma in a Rat Model. Ann Surg 2017;266:91-8.
5. C Cancer Genome Atlas Research Network; Analysis Working Group: Asan University; BC Cancer Agency; et al. Integrated genomic characterization of oesophageal carcinoma. Nature 2017;541:169-75.

6. Dulak AM, Stojanov P, Peng S, et al. Exome and wholegenome sequencing of esophageal adenocarcinoma identifies recurrent driver events and mutational complexity. Nat Genet 2013;45:478-86.

7. Prins MJ, Verhage RJ, Ruurda JP, et al. Over-expression of phosphorylated mammalian target of rapamycin is associated with poor survival in oesophageal adenocarcinoma: a tissue microarray study. J Clin Pathol 2013;66:224-8.

8. Shigaki H, Baba Y, Watanabe M, et al. PIK3CA mutation is associated with a favorable prognosis among patients with curatively resected esophageal squamous cell carcinoma. Clin Cancer Res 2013;19:2451-9.

9. Harada K, Baba Y, Shigaki H, et al. Prognostic and clinical impact of PIK3CA mutation in gastric cancer: pyrosequencing technology and literature review. BMC Cancer 2016;16:400.

10. Hildebrandt MA, Yang H, Hung MC, et al. Genetic variations in the $\mathrm{PI} 3 \mathrm{~K} / \mathrm{PTEN} / \mathrm{AKT} / \mathrm{mTOR}$ pathway are associated with clinical outcomes in esophageal cancer patients treated with chemoradiotherapy. J Clin Oncol 2009;27:857-71.

11. Saeed N, Shridhar R, Hoffe S, et al. AKT expression is associated with degree of pathologic response in adenocarcinoma of the esophagus treated with neoadjuvant therapy. J Gastrointest Oncol 2016;7:158-65.

12. Ohtsu A, Ajani JA, Bai YX, et al. Everolimus for previously treated advanced gastric cancer: results of the randomized, double-blind, phase III GRANITE-1 study. J Clin Oncol 2013;31:3935-43.

13. Al-Batran SE, Riera-Knorrenschild J, Pauligk C, et al. A randomized, double-blind, multicenter phase III study evaluating paclitaxel with and without RAD001 in patients with gastric cancer who have progressed after therapy with a fluoropyrimidine/platinum-containing regimen (RADPAC). J Clin Oncol 2017;35:4.

14. O'Reilly KE, Rojo F, She QB, et al. mTOR inhibition induces upstream receptor tyrosine kinase signaling and activates Akt. Cancer Res 2006;66:1500-8.

15. Stewart A, Thavasu P, de Bono JS, et al. Titration of signalling output: insights into clinical combinations of MEK and AKT inhibitors. Ann Oncol 2015;26:1504-10.

16. Zimmermann S, Moelling K. Phosphorylation and regulation of Raf by Akt (protein kinase B). Science 


$$
\text { 1999;286:1741-4. }
$$

17. Hoeflich KP, O'Brien C, Boyd Z, et al. In vivo antitumor activity of MEK and phosphatidylinositol 3-kinase inhibitors in basal-like breast cancer models. Clin Cancer Res 2009;15:4649-64.

18. Kopetz S, Desai J, Chan E, et al. Phase II Pilot Study of Vemurafenib in Patients With Metastatic BRAF-Mutated Colorectal Cancer. J Clin Oncol 2015;33:4032-8.

doi: $10.21037 /$ shc. 2017.10 .07

Cite this article as: Harada $\mathrm{K}$, Song S, Baba H, Ajani JA. Is phosphatidylinositol 3-kinase/AKT/mammalian target of rapamycin pathway therapeutic target for esophageal adenocarcinoma. Shanghai Chest 2017;1:51.
19. Mao M, Tian F, Mariadason JM, et al. Resistance to BRAF inhibition in BRAF-mutant colon cancer can be overcome with PI3K inhibition or demethylating agents. Clin Cancer Res 2013;19:657-67.

20. Deguchi Y, Okabe H, Oshima N, et al. PTEN loss is associated with a poor response to trastuzumab in HER2overexpressing gastroesophageal adenocarcinoma. Gastric Cancer 2017;20:416-27. 